\title{
Contribuição dos Métodos de Diagnóstico por Imagem na Avaliação da Espondilólise
}

\section{Contribution of the Imasing Methods in the Evaluation of Spondylolysis}

Cristiane Soares Zoner ${ }^{(1)}$, Denise Tokechi do Amaral ${ }^{(2)}$, Jamil Natour ${ }^{(2)}$, Artur da Rocha Correa Fernandes ${ }^{(2)}$

\section{INTRODUÇÃO}

Espondilólise é uma interrupção da pars articularis ou istmo ${ }^{(1)}$. A prevalência deste defeito foi relatada entre 2,3 e $10 \%$ da população geral, sendo que a maioria parece ser assintomática. Entretanto, cerca de $25 \%$ dos pacientes com este defeito podem apresentar dor lombar baixa ou dor radicular ${ }^{(2)}$. A prevalência parece ser aumentada em homens e indivíduos fortemente ativos ou em atletas. Há um forte componente de hereditariedade ${ }^{(3)}$. A maioria dos casos ocorre nos níveis L5 (cerca de 90\%) ${ }^{(2)}$. O defeito é unilateral em aproximadamente um quinto dos $\operatorname{casos}^{(3)}$.

A etiologia é controversa, com muitas teorias propostas. Porém, acredita-se que na maioria dos casos é uma alteração de desenvolvimento que ocorre na primeira década de vida. Fraturas de estresse durante a adolescência também são encontradas ${ }^{(4)}$.

Outras teorias, como alteração congênita e fratura aguda do istmo, foram propostas. Uma pequena porcentagem dos casos tem origem displásica ${ }^{(1)}$. Como esta lesão foi descrita apenas em humanos, é possível que a postura ereta associada a estresse inapropriado possa representar um fator etiológico importante. Provavelmente, estas diferentes origens estejam presentes sozinhas ou de maneira associada em cada caso.

As alterações relacionadas à espondilólise podem ser reconhecidas pela radiografia, tomografia computadorizada (TC), ressonância magnética (RM) e cintilografia. Os achados que podem ser encontrados e as vantagens e desvantagens de cada método serão discutidos a seguir.

Palavras-chave: coluna vertebral, espondilólise, imagem, ressonância magnética, tomografia computadorizada, raios $\mathrm{X}$.

\section{RADIOGRAFIA}

A alteração é vista à radiografia no istmo ou pars articularis, uma região da lâmina entre as facetas articulares superior e inferior (Figuras 1, 2 e 3). O sinal radiológico mais precoce é a osteopenia, seguida pelo calo endosteal. A linha de fratura pode não ser evidente, sendo que neste caso o achado dominante é esclerose. O perfil, geralmente, demonstra o defeito, especialmente se a espondilolistese estiver associada ${ }^{(3)}$. Enquanto as não-uniões crônicas podem ser demonstradas à radiografia, ela, entretanto, é pouco confiável para detecção de lesões precoces e agudas ${ }^{(4)}$.

A incidência ântero-posterior demonstra o defeito da pars interarticularis apenas em uma pequena proporção dos casos, embora possam ser vistos sinais secundários como fragmentação laminar, espessamento laminar e mau

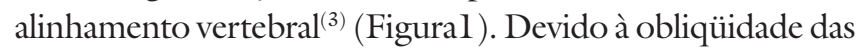
vértebras, a pars interarticularis na região lombar baixa não é adequadamente avaliada na incidência frontal, justamente onde a freqüência de lise é maior. Uma incidência ântero-

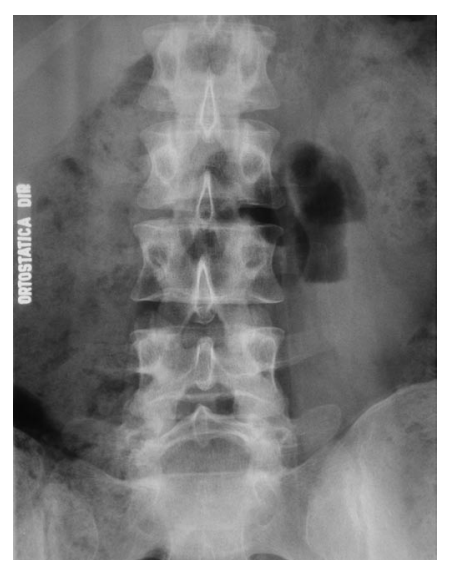

Figura 1 - Radiografia AP evidencia defeito na pars interarticularis direita de L5.

1. Departamento de Diagnóstico por Imagem da Universidade Federal de São Paulo (UNIFESP).

2. Disciplina de Reumatologia da UNIFESP.

Endereço para Correspondência: Artur da Rocha Fernandes, Departamento de Diagnóstico por Imagem da UNIFESP, Rua Botucatu, 740, CEP 04039-002,

São Paulo, SP, Brasil. 
posterior com angulação cranial de 30 graus foi proposta para permitir uma avaliação adequada dos istmos nesta região, permitindo uma visão praticamente frontal dos mesmos, com mínima distorção, encurtamento ou sobreposição dos corpos vertebrais ${ }^{(7)}$.

A radiografia oblíqua mostra, em grande parte das vezes, o defeito radiotransparente no istmo. Lachapèle descreveu a aparência do arco neural e seus processos como um "cachorrinho". O defeito parece como uma radiotransparência linear irregular, que foi chamada de “coleira do cachorrinho (Figura 2). A margem óssea pode ser esclerótica e irregular ${ }^{(1)}$.

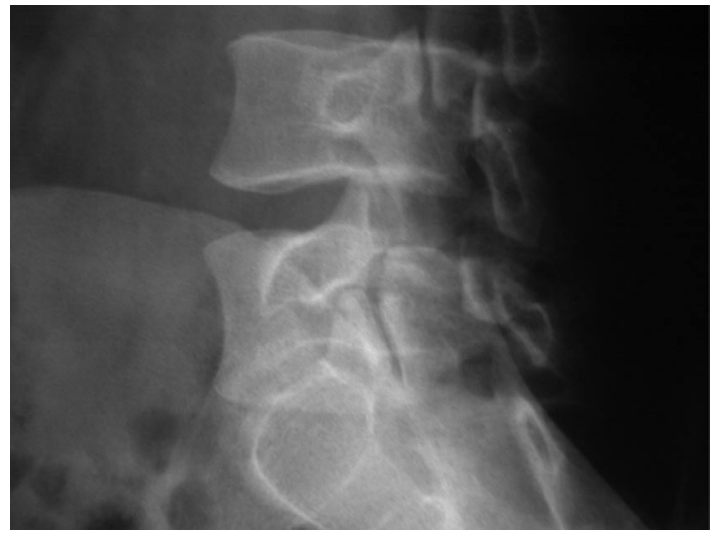

Figura 2 - Radiografia oblíqua demonstra o defeito no pescoço do "cachorrinho de Lachapèle", que corresponde ao istmo de L5.

A incidência colimada em perfil foi a mais sensível para detecção do defeito da pars articularis em um estudo comparando incidências ântero-posterior, ântero-posterior com angulação cranial, oblíquas, perfil e perfil colimada ${ }^{(3)}$ (Figura 3).

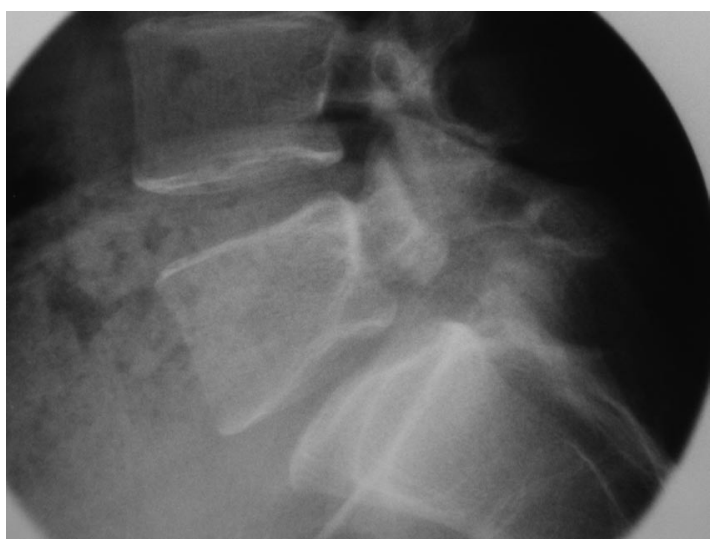

Figura 3 - Radiografia localizada em perfil demonstrando o defeito na pars interarticularis de L5, com espondilolistese anterior grau I.
A definição de instabilidade em espondilólise é controversa e pobremente definida. Alguns autores definem como mobilidade excessiva, outros consideram que a coluna precisa ser instável para haver espondilolistese ${ }^{(3)}$. A espondilolistese é o deslizamento de uma vértebra superior em relação à inferior. Classicamente, este termo é utilizado quando o deslocamento é anterior. Foi classificada em categorias por Meyerding. O grau 1 corresponde ao deslizamento anterior da vértebra superior até um quarto da dimensão do planalto da vértebra inferior, em uma visão em perfil. O grau aumenta para cada quarto da dimensão ântero-posterior do planalto vertebral que é descoberto pela vértebra superior (Figura 4). O grau 5 ou espondiloptose é o grau mais acentuado, quando há um deslocamento completo da vértebra superior, que se situa anteriormente à vértebra inferior ${ }^{(3)}$ (Figura 5 ). Há outras causas de espondilolistese que variam de displásica até degenerativa.

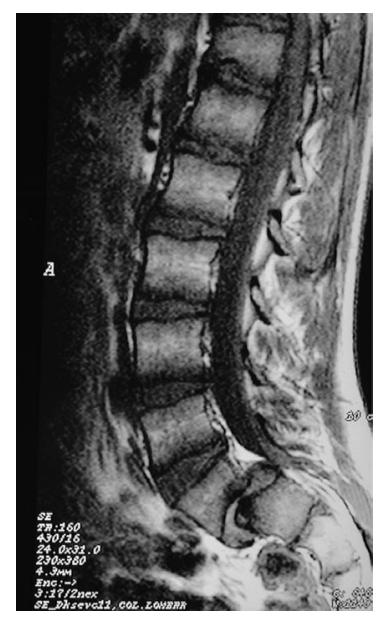

Figura 4 - RM ponderada em T1 no plano sagital evidencia espondilolistese grau II de Meyerding.
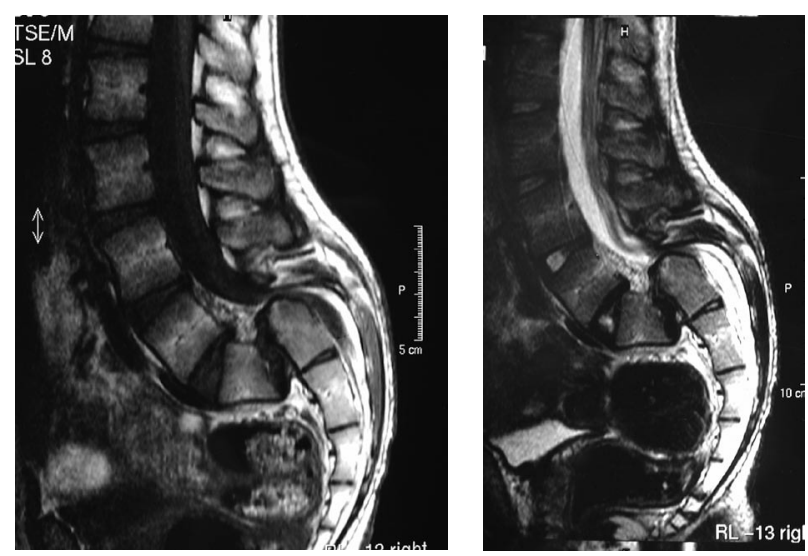

Figura 5 - RM ponderadas em T1 e T2 evidenciam espondilolistese grau $\mathrm{V}$ de Meyerding ou espondilotose. 
Para avaliar a instabilidade, radiografias em perfil com flexão e extensão em posição ortostática podem ser realizadas. Apesar da controvérsia em relação à instabilidade, qualquer deslocamento adicional do segmento com listese de $4 \mathrm{~mm}$ ou diferença na mobilidade angular acima de 11 graus em resposta a flexão e extensão é considerada com prognóstico pobre ao tratamento conservador ${ }^{(1)}$.

\section{TOMOGRAFIA COMPUTADORIZADA}

A tomografia computadorizada (TC) é, provavelmente, o melhor método para demonstrar os defeitos da pars interarticularis e também pode ser utilizada para avaliar consolidação ${ }^{(4)}$. Os cortes axiais devem incluir o plano acima do forame neural para permitir a demonstração do defeito. Neste plano, o aspecto normal é de um arco intacto, composto pelo muro posterior do corpo vertebral, as paredes mediais dos pedículos, a pars interarticularis ântero-medial, a lâmina e a margem anterior do processo espinhoso. Nos casos de lise, um "arco incompleto" é encontrado (Figura 6 A, B e C). O plano descrito corresponde usualmente ao nível que as veias basivertebrais emergem do corpo vertebral, servindo como um marcador da posição esperada do $\operatorname{arco~intacto~}^{(3)}$.
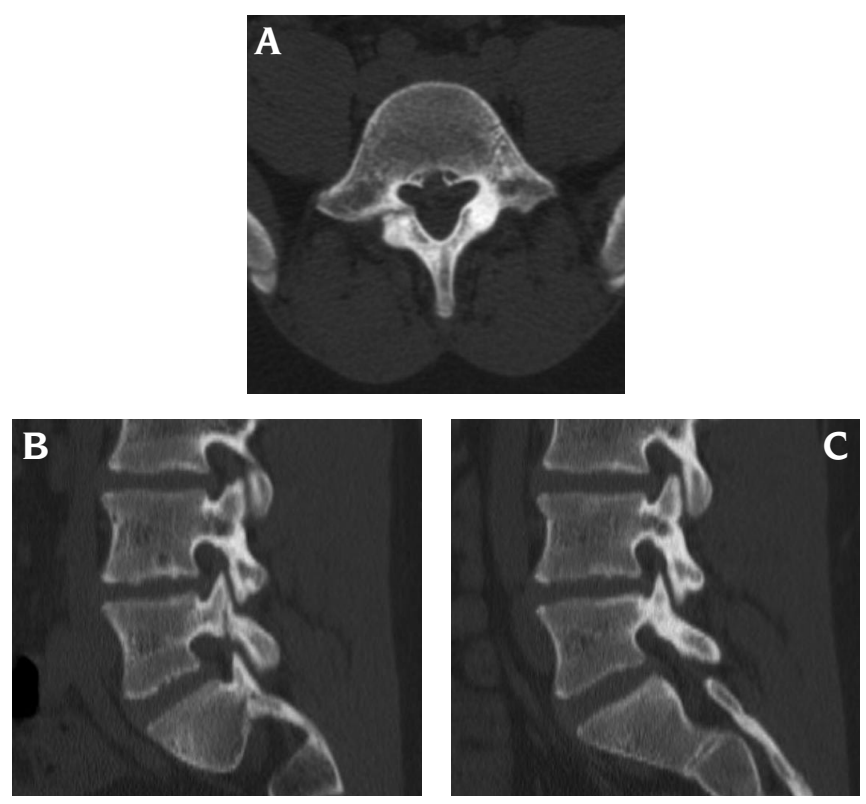

Figura 6 A, B e C - TC demonstra o defeito da pars interarticularis de L5, 0 aspecto da visão no plano axial e parassagital.

Os defeitos da pars interarticularis podem ser diferenciados das facetas articulares pela ausência de margens subarticulares densas, a presença de irregularidade ou fragmentação das margens do defeito, a ausência das incisuras normais encontradas junto às facetas que servem de local de inserção das cápsulas articulares e a orientação da lise, que geralmente é mais horizontal do que orientação levemente oblíqua das facetas ${ }^{(3)}$.

O deslocamento anterior do corpo vertebral, combinado ao deslocamento posterior do arco posterior, promove um aumento do diâmetro ântero-posterior do canal vertebral (Figura 7), sendo que nos casos mais graves, um aspecto de "canal duplo" pode ser encontrado.

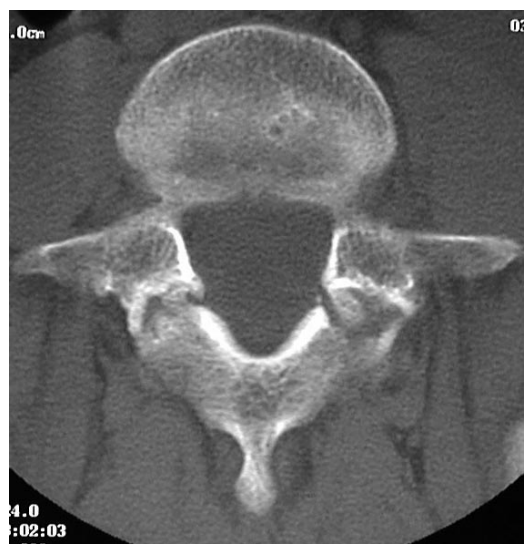

Figura 7 - TC mostra lise acompanhada do sinal do alargamento do canal no diâmetro ântero-posterior.

O disco intervertebral pode apresentar um "pseudoabulamento", que representa a projeção do ânulo fibroso posterior à vértebra com listese anterior.

Por vezes, a avaliação apenas no plano axial pode deixar dúvidas. A TC com gantry invertido foi utilizada para identificar os defeitos em um plano próximo do coronal, demonstrando a descontinuidade dos elementos posteriores da vértebra no aparelho convencional axial. Atualmente, os tomógrafos helicoidais e, principalmente, os multidetectores, permitem reconstruções em outros planos com alta resolução, permitindo o diagnóstico com facilidade, pelo fato do plano sagital permitir uma melhor avaliação da pars interarticularis (Figura $6 \mathrm{~B}$ e C).Entretanto, a TC não pode distinguir entre lesões ativas e não-ativas ${ }^{(4)}$.

\section{RESSONÂNCIA MAGNÉTICA}

O sinal mais confiável de pars interarticularis normal à RM é a continuidade da intensidade de sinal da medular óssea, através da pars interarticularis, principalmente, nas seqüências ponderadas em Tl. Entretanto, este aspecto é visualizado somente em $30-66 \%$ dos $\operatorname{casos}^{(5)}$.

Já o sinal primário da espondilólise à RM é a interrupção das margens corticais e do sinal da medular óssea da pars interarticularis. Porém, o diagnóstico pode ser difícil devido a fatores como obliquiidade da direção principal da 
pars interarticularis em relação aos planos sagital e transversal. Alterações escleróticas com perda do sinal da medular óssea podem ser causadas pelas alterações degenerativas das facetas articulares adjacentes e efeito de volume parcial das partes moles circunjacentes. Por isso, sinais indiretos têm sido usa-

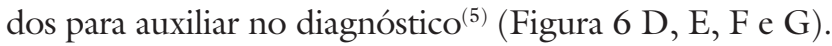
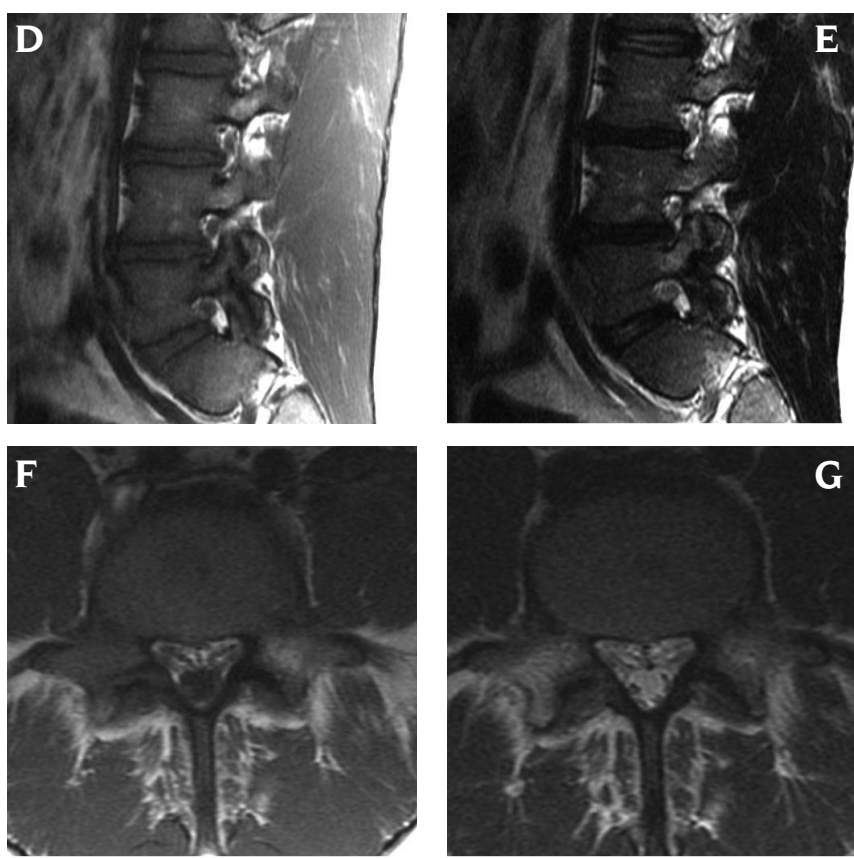

Figura 6 D, E, F e G - RM ponderada em T1 e T2 nos planos parassagital e axial evidenciam a linha de hipossinal na pars interarticularis com sinal com padrão de edema na medular óssea do pedículo adjacente.

Os sinais indiretos são: aumento do diâmetro ânteroposterior do canal vertebral no nível do defeito do istmo, acunhamento da porção posterior do corpo vertebral e alteração reacional da intensidade de sinal dos pedículos.

$\mathrm{O}$ aumento do diâmetro ântero-posterior do canal no nível do defeito pode ser visto tanto pelo deslocamento anterior do corpo vertebral em relação aos elementos posteriores quanto pelo deslocamento posterior do arco neural relativo aos arcos das vértebras acima e abaixo ${ }^{(6)}$.

A alteração do sinal da medular óssea dos pedículos pode ser visualizada em uma proporção significativa de casos de espondilólise (cerca de 40\%) (Figura 6 e 8 ), sendo semelhantes às alterações descritas por Modic para as alterações degenerativas dos planaltos vertebrais ${ }^{(2)}$. O tipo mais freqüentemente encontrado é a conversão gordurosa, caracterizada por hipersinal em T1 e T2 ${ }^{(2)}$ (Figura 8). Uma área com padrão de edema na pars interarticularis com hipossinal em T1 e hipersinal em T2, que pode indicar a presença de estresse ativo na pars e representar um precur- sor do desenvolvimento de espondilólise, mesmo quando a radiografia simples é normal ${ }^{(1)}$.

Foi proposto, também, o sinal da interposição da gordura epidural entre a dura-máter e o processo espinhoso ${ }^{(5)}$ (Figura 8), que representa um sinal qualitativo do aumento do diâmetro ântero-posterior do canal vertebral, em casos em que a espondilolistese não é evidente. Nos casos normais, há contato entre a dura-máter e o processo espinhoso. A presença deste sinal foi encontrada apenas nos casos com espondilólise ou lipomatose epidural, cujo diagnóstico pode ser feito através de outros achados. Porém, a falta deste sinal não indica ausência de lise.
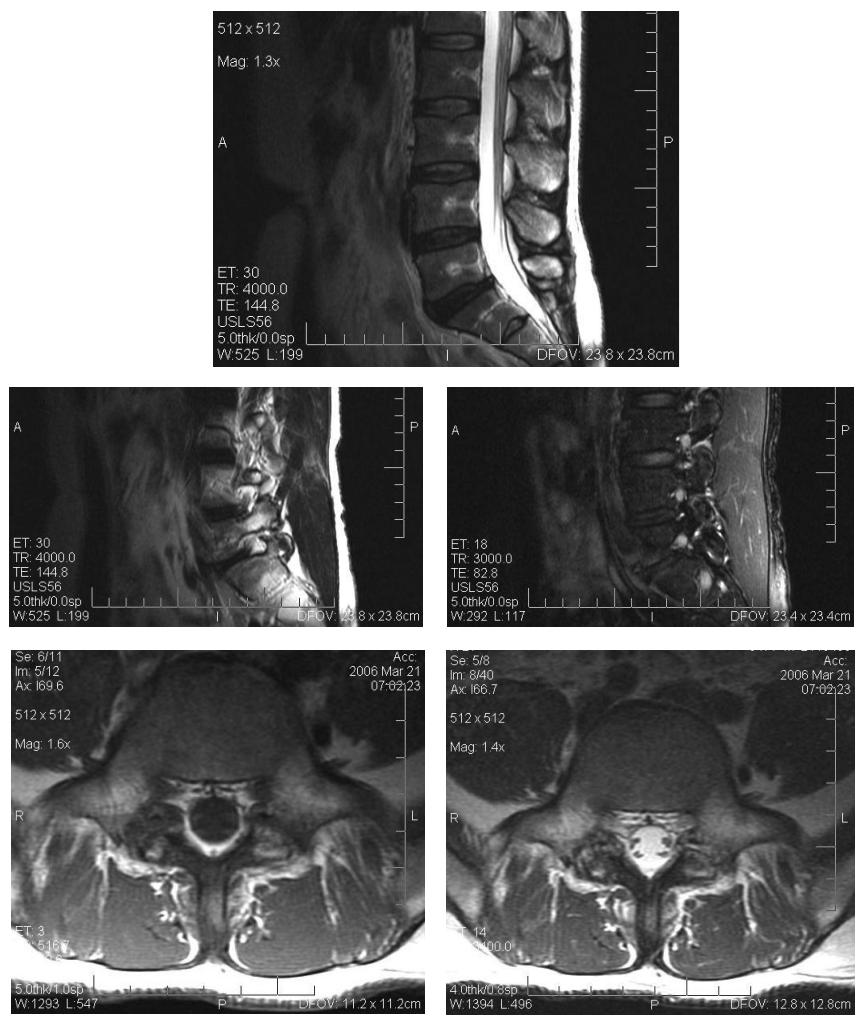

Figura 8 - RM ponderadas em T1 e T2 com supressão de gordura nos planos sagital e axial. Nota-se o sinal da interposição da gordura entre a dura mater e o processo espinhoso de $\mathrm{L} 5$ no plano sagital próximo a linha média. 0 defeito da pars interarticularis pode ser observado nos planos parassagital e axial com linha de hipossinal em todas as sequiências. Observa-se também a alteração de sinal do pedículo com padrão de gordura caracterizado por hipersinal em T1 e T2 sem supressão de gordura e hipossinal em T2 com supressão.

As imagens parassagitais da RM permitem avaliar o forame intervertebral, a posição das raízes nervosas e a presença de encarceramento da raiz ${ }^{(1)}$. O forame intervertebral envolvido nos casos de espondilólise com espondilolistese torna-se mais horizontal, com um aspecto bilobulado $^{(6)}$. A obliteração ou perda quase completa do 
alto sinal em Tl da gordura que, normalmente, margeia ou circunda a raiz nervosa pode sugerir encarceramento. A estenose do forame tem um padrão específico, sendo o recesso superior do forame intervertebral ocupado. Esta alteração pode ter repercussão clínica, com sintomas neurológicos de compressão radicular, visto que é a localização habitual da raiz nervosa.

Campbell et a ${ }^{(4)}$ concluíram que a RM é um método acurado para demonstrar a pars interarticularis normal, defeitos completos agudos e defeitos crônicos estabelecidos, porém apresenta limitações em diagnosticar com acurácia reação de estresse e defeitos incompletos. Estes autores destacam a importância da distinção entre fraturas completas e incompletas, visto que as fraturas incompletas cicatrizam espontaneamente com freqüência, enquanto há uma alta incidência de não-união para defeitos completos agudos.

A RM tem a vantagem adicional de ser útil para determinar que outras anormalidades contribuem de forma mais provável para a dor lombar.

\section{MEDICINA NUCLEAR}

A espondilólise apresenta um aspecto não-específico à cintilografia óssea, com o diagnóstico final dependendo de outros métodos de imagem, porém pode fornecer informações a respeito da atividade metabólica do istmo, permitindo, desta forma, uma avaliação funcional ${ }^{(3)}$. Assim, uma lesão de estresse aguda da pars interarticularis pode não ser aparente à radiografia e evidenciada pela cintilografia, enquanto o oposto pode ocorrer em locais de espondilólise de longa data que não estão metaboli-

\section{REFERÊNCIAS}

1. Yochum TR, Rowe LJ: Essentials of Skeletal Radiolçogy, $2^{\text {nd }}$ ed, University Graphics Inc: 327-356.

2. Ulmer JL, Elster AD, Mathews VP, Allen AM: Lumbar Spondylolysis: Reactive Marrow Changes Seen in Adjacente Pedicles on MRI Images. AJR 164: 429-433, 1995.

3. Resnick D: Diagnosis of Bone and Joint Disorders, $3^{\text {rd }}$ ed, California, WB Saunders Company, v.5 : 3003-3006, 1995.

4. Campbell RSD, Grainger AJ, Hide IG, Papastefanou S, Greenough CG: Juvenile Spondylolysis: a comparative analysis of CT, SPECT and MRI. Skeletal Radiology 34: 63-73, 2005. camente ativas.

O SPECT é o método mais sensível para localizar remodelação óssea em uma área de anatomia complexa e permite a diferenciação entre o acúmulo do radiotraçador no arco neural e no corpo vertebral ${ }^{(1)}$. Apresenta um papel na avaliação da atividade fisiológica da pars em pacientes sintomáticos e podem auxiliar em decisões terapêuticas, como, por exemplo, a necessidade de reduzir a atividade física. Porém, também apresenta limitações, devido à baixa especificidade e visto que estudos positivos foram relatados em sítios não-dolorosos em atletas, sugerindo uma resposta de estresse fisiológica e adaptação ${ }^{(4)}$.

\section{CONCLUSÃO}

A avaliação em casos de suspeita de espondilólise pode ser iniciada com a radiografia simples. Confirmando-se a suspeita, pode-se prosseguir com RM ou cintilografia para se obter informações funcionais. Em casos em que a radiografia inicial é duvidosa, pode-se prosseguir a investigação também com RM, visto que ela pode possibilitar ou excluir o diagnóstico, além de fornecer outras informações a respeito da provável causa da dor lombar. A TC pode ser utilizada quando a RM é duvidosa quanto à presença de lise ou quando há necessidade de estudar as alterações ósseas, como, por exemplo, a evolução da cicatrização e união óssea. Por último, as radiografias dinâmicas em flexão e extensão permitem melhor caracterização da instabilidade e fornecem dados que podem ter importância na conduta terapêutica.

Declaramos a inexistência de conflitos de interesse.
5. Sherif H, Mahfouz AE: Epidural fat interposition between dura mater and Spinous Process: a New Sign for the Diagnosis of Spondylolysis on MR Imaging of the Lumbar Spine. European Radiology 14: 970-973, 2004.

6. Jinkins JR, Matthes JC, Sener RN, Venkatappan S, Rauch R: Spondylolysis, Spondylolysthesis and Associated Nerve Root Entrapment in the Lumbosacral Spine: MR Evaluation. AJR 159: 799-803, 1992.

7. Libson E, Bloom RA: Anteroposterior Angulatede view: a New Radiographic Technique for the Evaluation of Spondylolysis. Radiology 183: 315-316, 1983. 